\title{
Anti-complementary Activity of Triterpenoides from Fruits of Zizyphus jujuba
}

\author{
Sang-Myung LeE, ${ }^{a}$ Jin-Gyu PArK, ${ }^{a}$ You-Hee LeE,${ }^{a}$ Cheal-Gyu Lee, ${ }^{b}$ Byung-Sun Min, $*, c$ \\ Jung-Hee KIM, ${ }^{c}$ and Hyeong-Kyu $\mathrm{LEE}^{c}$ \\ ${ }^{a}$ KT\&G Central Research Institute; 302, Shinseng-dong, Yuseong, Daejeon, 305-805, Korea: ${ }^{b}$ Department of \\ Environmental Engineering, Chongju University; Chongju 360-764, Korea: and ${ }^{c}$ Laboratory of Immunomodulator, Korea \\ Research Institute of Bioscience and Biotechnology; Daejeon, 305-333, Korea. \\ Received May 31, 2004; accepted July 28, 2004
}

In order to determine on the anti-complement activity of triterpenes, following eleven triterpenoides were isolated from the fruits of the Zizyphus jujuba MILL: ceanothane-type triterpenes: colubrinic acid (1), zizyberenalic acid (11); lupane-type triterpenes: alphitolic acid (2), 3-O-cis-p-coumaroyl alphitolic acid (3), 3-O-trans-pcoumaroyl alphitolic acid (4), betulinic acid (7), betulonic acid (9); and oleanane-type triterpenes: 3-O-cis-pcoumaroyl maslinic acid (5), 3-O-trans-p-coumaroyl maslinic acid (6), oleanolic acid (8), oleanonic acid (10). These compounds were examined for their anti-complement activity against the classical pathway of the complement system. Among them, compounds 5, 6, and 8 exhibited significant anti-complement activity with $\mathrm{IC}_{50}$ values of 101.4, 143.9, and $163.4 \mu \mathrm{M}$, respectively, whereas the ceanothane-type and the lupane-type triterpenes were inactive. This suggests that the oleanane-structure plays an important role in inhibiting the hemolytic activity of human serum against erythrocytes.

Key words anti-complementary activity; Zizyphus jujuba; oleanane-type triterpene; coumaroyl group

The complement system is a major effector of humoral immunity and is activated by a cascade mechanism via an antigen-antibody mediated process (classical pathway, CP), an antibody independent process (alternative pathway, AP), or through mannan binding lectin/MBL-associated serine protease (MBL/MASP). ${ }^{1)}$ The proteolytic cascade allows for a very high amplification rate, which in the next step activates the enzymes later in the cascade. This in turn cleaves the non-enzymes, such as C3, C4, and C5. The pathway converges the $\mathrm{C} 3$ convertase step leading to $\mathrm{C} 5$ convertase and the self-assembly of the membrane attack complex (MAC). In complement activation, the complement components induce the release of mediators from the mast cells and lymphocytes, causing a variety of diseases (i.e., rheumatoid arthritis, osteoarthritis, atopic dermatitis, lung fluid inflamatio, and atherosclerotic lesion), as well as can be fated if occurring after an organ transplantation. ${ }^{2)}$ This effect is normally beneficial for the host, but can also cause adverse effects depending on the site as extent and duration of complement activation. The modulation of complement activity can be important to the treatment of inflammation.

During the screening of plant extracts, the complement inhibiting property of an EtOAc-soluble fraction of the fruit of Zizyphus jujuba MiLl was examined. This plant is a thorny rhamnaceous plant that is widely distributed in Europe and Southeastern Asia. The seed, which known as Suan Tsao Zen in China, is a famous Chinese medicine that is used for the treating insomnia. ${ }^{3)}$ A literature survey shows that this plant is rich in biological active compounds, such as triterpenes, cyclopeptide alkaloids and flavonoids, ${ }^{4-10)}$ which have been shown to have inhibitory effects on histamine release, ${ }^{11)}$ hippocampal formation, ${ }^{12)}$ cycolooxygenase- 1 and $-2,{ }^{13)}$ activation activity of choline acetyltransferase, ${ }^{14)}$ cytotoxic activity, ${ }^{15)}$ and immunological adjuvant activity. ${ }^{16)}$

It was previously reported that the oleanane-type triterpene saponins, such as ginseng saponins from Korean red ginseng, ${ }^{17)}$ kaikasaponins and soyasaponins from Pueraria lobata ${ }^{18)}$ bisdesmosidic saponins from Tiarella polyphylla, ${ }^{19}$ ) and hederagenin saponins from Dipsacus asper ${ }^{20)}$ have significant anti-complement activity. In addition, lanostane-type triterpenes from Ganoderma lucidum, ${ }^{21)}$ prostane-type triterpenes, ${ }^{22}$ and dammarane-type saponins ${ }^{17}$ inhibited the hemolytic activity of the complement system. Kim et al. reported that the oleanane-type saponins have a more potent anti-complement activity than that of the dammarane-type saponins. ${ }^{17)}$ Therefore, a greater knowledge of the anti-complement activity of the coumaroyl moiety on oleanane-type triterpene and the lupane-type triterpenes will enable a better understand of biological triterpenes. Accordingly, this paper reports the anti-complementary activities of eleven triterpenes isolated from the fruit of Z. jujuba.

\section{MATERIALS AND METHODS}

General Experimental Procedures Melting point was measured on a Yanagimoto micro hot-stage melting point apparatus and was uncorrected. Optical rotations were measured with a JASCO DIP-370 digital polarimeter. ${ }^{1} \mathrm{H}$ - and ${ }^{13} \mathrm{C}-\mathrm{NMR}$ spectra were recorded on Bruker AMX-300 or AMX-500 spectrometers. Chemical shifts were presented as ppm. ESIMASS were measured on a HP5989A DIP mass spectrometer.

Plant Fruits of Zizyphus jujuba were purchased from an oriental medicine market in Kumsan, Korea. A voucher specimen (KT\&G 0005) was deposited in the herbarium of the KT\&G Central Research Institute.

Isolation of Triterpenoids The fruits of Z. jujuba $(1 \mathrm{~kg})$ were extracted with $\mathrm{MeOH}(3 \mathrm{l})$ at room temperature $(7 \mathrm{~d} \times 3)$ to yield an extract $(400 \mathrm{~g})$. The $\mathrm{MeOH}$ extract was suspended in $\mathrm{H}_{2} \mathrm{O}(11)$ and was then shaken with EtOAc $(11 \times 2$, each time, $75 \mathrm{~g})$, and $\mathrm{BuOH}$ saturated with $\mathrm{H}_{2} \mathrm{O}(11 \times 3$, each time, $200 \mathrm{~g}$ ), successively. A portion of EtOAc-soluble fraction 
(3 g) was divided into ten fractions (Fr. 1-Fr. 10; $40 \mathrm{ml}$ each) on Sep-Pak RP-C18 Cartridges (5 G) eluted with acetonitrile $0 \rightarrow 100 \%$ (each fraction was increased by $10 \%$ with acetonitrile). The Frs. 7 and $8(500 \mathrm{mg})$ were preparativeHPLC on YMC-Pak ODS-AQ column $(300 \times 10 \mathrm{~mm}$ I.D.) eluted with aqueous acetonitrile $(75 \%, 1.5 \mathrm{ml} / \mathrm{min})$ to give fifteen sub-fractions (Fr. 1'-Fr. 15'; each $10 \mathrm{ml}$ ). These subfractions were further purified on preparative HPLC eluting with $75 \%$ aqueous acetonitrile to yield compounds $\mathbf{1}-\mathbf{1 1}$ (Fig. 1). Compounds: 1 ( $t_{\mathrm{R}}: 14.6 \mathrm{~min}, 3 \mathrm{mg}$; from Fr. $\left.2^{\prime}\right), 2$ $\left(t_{\mathrm{R}}: 16.6 \mathrm{~min}, 11 \mathrm{mg}\right.$; from Fr. $\left.2^{\prime}\right), 3\left(t_{\mathrm{R}}: 19.8 \mathrm{~min}, 8 \mathrm{mg}\right.$; from Fr. $\left.3^{\prime}\right), 4\left(t_{\mathrm{R}}: 20.4 \mathrm{~min}, 7 \mathrm{mg}\right.$; from Fr. $\left.3^{\prime}\right), 5\left(t_{\mathrm{R}}: 21.2 \mathrm{~min}\right.$, $2 \mathrm{mg}$; from Fr. $\left.3^{\prime}\right), 6\left(t_{\mathrm{R}}: 22.4 \mathrm{~min}, 1 \mathrm{mg}\right.$; from Fr. $\left.3^{\prime}\right), 7$ $\left(t_{\mathrm{R}}: 22.8 \mathrm{~min}, 17 \mathrm{mg}\right.$; from Fr. $\left.4^{\prime}, 5^{\prime}\right), 8\left(t_{\mathrm{R}}: 24.3 \mathrm{~min}, 3 \mathrm{mg}\right.$; from Fr. $\left.4^{\prime}, 5^{\prime}\right), 9\left(t_{\mathrm{R}}: 26.9 \mathrm{~min}, 9 \mathrm{mg}\right.$; from Fr. $\left.6^{\prime}\right), \mathbf{1 0}$ $\left(t_{\mathrm{R}}: 27.7 \mathrm{~min}, 7 \mathrm{mg}\right.$; from Fr. $\left.6^{\prime}, 7^{\prime}\right), \mathbf{1 1}\left(t_{\mathrm{R}}: 29.1 \mathrm{~min}, 7 \mathrm{mg}\right.$; from Fr. $8^{\prime}$ ).

Colubrinic Acid (1): White needles (MeOH); mp 262-

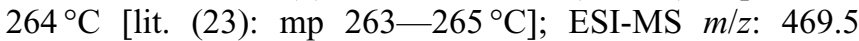
$[\mathrm{M}-\mathrm{H}]^{-}, 493.6[\mathrm{M}+\mathrm{Na}]^{+} ;[\alpha]_{\mathrm{D}}^{18}:+3.0^{\circ}(c=0.5$, pyridine $)$. ${ }^{1} \mathrm{H}-\mathrm{NMR}\left(500 \mathrm{MHz}\right.$, pyridine- $\left.d_{5}\right) \delta: 10.12(\mathrm{~d}, J=4.4 \mathrm{~Hz}, \mathrm{H}-$ 2), 4.90, 4.77 (each br s, H-30), 4.65 (d, $J=8.6 \mathrm{~Hz}, \mathrm{H}-3$ ), 3.42 (dt, $J=3.8,11.2 \mathrm{~Hz}, \mathrm{H}-19), 2.55$ (dd, $J=4.4,8.6 \mathrm{~Hz}, \mathrm{H}-1)$, 1.79 (s, H-29). ${ }^{13} \mathrm{C}-\mathrm{NMR}\left(125 \mathrm{MHz}\right.$, pyridine- $\left.d_{5}\right) \delta: 206.0$ (C-2), 179.0 (C-28), 151.4 (C-20), 110.3 (C-30), 80.8 (C-3), 73.7 (C-1), 19.3 (C-29).

Alphitolic Acid (2): Colorless needles $\left(\mathrm{CHCl}_{3}-\mathrm{MeOH}\right)$; mp 275-278 ${ }^{\circ} \mathrm{C}$; ESI-MS $m / z$ : $471.4[\mathrm{M}-\mathrm{H}]^{-}, 495.6$ $[\mathrm{M}+\mathrm{Na}]^{+} ; \quad[\alpha]_{\mathrm{D}}^{19}:-4.0^{\circ} \quad(c=1.0, \quad$ pyridine $) .{ }^{1} \mathrm{H}-\mathrm{NMR}$ $\left(300 \mathrm{MHz}, \mathrm{CDCl}_{3}\right) \delta: 4.76,4.89$ (each d, $J=2 \mathrm{~Hz}, \mathrm{H}-30$ ), $4.11(\mathrm{~m}, \mathrm{H}-2), 3.36$ (d, $J=10.0 \mathrm{~Hz}, \mathrm{H}-3) .{ }^{13} \mathrm{C}-\mathrm{NMR}(75 \mathrm{MHz}$, $\left.\mathrm{CDCl}_{3}\right) \delta: 48.3(\mathrm{C}-1), 84.1$ (C-3), 68.7 (C-2).

3-O-(cis-p-Coumaroyl)-alphitolic Acid (3): White powder; mp 208-210 ${ }^{\circ} \mathrm{C}$; ESI-MS $m / z: 617.6[\mathrm{M}-\mathrm{H}]^{-} ;[\alpha]_{\mathrm{D}}^{20}:+40^{\circ}$ ( $c=1.0$, pyridine). ${ }^{1} \mathrm{H}-\mathrm{NMR}\left(500 \mathrm{MHz}, \mathrm{CD}_{3} \mathrm{OD}\right)$ : coumaroyl moiety $\delta: 5.85\left(\mathrm{~d}, J=12.2 \mathrm{~Hz}, \mathrm{H}-2^{\prime}\right), 6.88(\mathrm{~d}, J=12.2 \mathrm{~Hz}$, H-3') 6.91 (d, $\left.J=8.2 \mathrm{~Hz}, \mathrm{H}-3^{\prime \prime}, 5^{\prime \prime}\right), 7.73$ (d, $J=8.2 \mathrm{~Hz}$, $\left.\mathrm{H}-2^{\prime \prime}, 6^{\prime \prime}\right)$.

3-O-(trans-p-Coumaroyl)-alphitolic Acid (4): White powder; mp 279-280 ${ }^{\circ} \mathrm{C}$; ESI-MS $m / z: 617.6[\mathrm{M}-\mathrm{H}]^{-}, 641.7$ $[\mathrm{M}+\mathrm{Na}]^{+} ;[\alpha]_{\mathrm{D}}^{25}:-33.1^{\circ}(c=0.8$, pyridine $) .{ }^{1} \mathrm{H}-\mathrm{NMR}(500$ $\left.\mathrm{MHz}, \mathrm{CD}_{3} \mathrm{OD}\right)$ : coumaroyl moiety $\delta: 6.30(\mathrm{~d}, J=16.4 \mathrm{~Hz}$, H-2') 7.66 (d, $\left.J=16.4 \mathrm{~Hz}, \mathrm{H}-3^{\prime}\right), 6.90$ (d, $J=8.2 \mathrm{~Hz}, \mathrm{H}-3^{\prime \prime}$, $\left.5^{\prime \prime}\right), 7.35$ (d, $\left.J=8.2 \mathrm{~Hz}, \mathrm{H}-2^{\prime \prime}, 6^{\prime \prime}\right)$.

3-O-(cis-p-Coumaroyl)-maslinic Acid (5): White powder; mp $190-194^{\circ} \mathrm{C}$; ESI-MS $m / z: 617.6[\mathrm{M}-\mathrm{H}]^{-}, 641.7$ $[\mathrm{M}+\mathrm{Na}]^{+} ;[\alpha]_{\mathrm{D}}^{24}:+9.1^{\circ}(c=1.1$, pyridine $) .{ }^{1} \mathrm{H}-\mathrm{NMR}(500$ $\left.\mathrm{MHz}, \mathrm{CD}_{3} \mathrm{OD}\right)$ : coumaroyl moiety $\delta: 5.83(\mathrm{~d}, J=12.2 \mathrm{~Hz}$, H-2'), 6.34 (d, J=12.2 Hz, H-3'), 6.87 (d, $J=8.2 \mathrm{~Hz}, \mathrm{H}-3^{\prime \prime}$, $\left.5^{\prime \prime}\right), 7.66\left(\mathrm{~d}, J=8.2 \mathrm{~Hz}, \mathrm{H}-2^{\prime \prime}, 6^{\prime \prime}\right)$.

3-O-(trans-p-Coumaroyl)-maslinic Acid (6): White powder; mp 278-282 ${ }^{\circ} \mathrm{C}$; ESI-MS $m / z: 617.6[\mathrm{M}-\mathrm{H}]^{-}, 641.7$ $[\mathrm{M}+\mathrm{Na}]^{+} ;[\alpha]_{\mathrm{D}}^{20}:+0.9^{\circ}(c=1.0$, pyridine $) .{ }^{1} \mathrm{H}-\mathrm{NMR}(500$ $\left.\mathrm{MHz}, \mathrm{CD}_{3} \mathrm{OD}\right)$ : coumaroyl moiety $\delta: 6.29(\mathrm{~d}, J=16.2 \mathrm{~Hz}, \mathrm{H}-$ $2^{\prime}$ ), 7.63 (d, $\left.J=16.2 \mathrm{~Hz}, \mathrm{H}-3^{\prime}\right), 6.91$ (d, $J=8.2 \mathrm{~Hz}, \mathrm{H}-3^{\prime \prime}, 5^{\prime \prime}$ ), 7.38 (d, $\left.J=8.2 \mathrm{~Hz}, \mathrm{H}-2^{\prime \prime}, 6^{\prime \prime}\right)$.

Betulonic Acid (9): White powder; $\mathrm{mp} 253-255^{\circ} \mathrm{C}$; ESIMS $m / z: 453.5[\mathrm{M}-\mathrm{H}]^{-}, 477.5[\mathrm{M}+\mathrm{Na}]^{+} ;[\alpha]_{\mathrm{D}}^{20}:+7.0^{\circ}$ ( $c=1.0$, pyridine). Identified by direct comparison of the ${ }^{1} \mathrm{H}$ NMR with an authentic sample, which was synthesized from betulinic acid by Jones oxidation.
Oleanonic Acid (10): White powder; mp 226-229 ${ }^{\circ} \mathrm{C}$; ESI-MS $m / z: 453.6[\mathrm{M}-\mathrm{H}]^{-}, 477.5[\mathrm{M}+\mathrm{Na}]^{+} ;[\alpha]_{\mathrm{D}}^{18}$ : $+64.2^{\circ}(c=1.0, \mathrm{MeOH})$. Identified by direct comparison of the ${ }^{1} \mathrm{H}-\mathrm{NMR}$ ) with an authentic sample, which was synthesized from oleanolic acid by Jones oxidation.

Zizyberenalic Acid (11): White powder; mp 214-216 ${ }^{\circ} \mathrm{C}$; ESI-MS $m / z: 451.5[\mathrm{M}-\mathrm{H}]^{-}, 475.5[\mathrm{M}+\mathrm{Na}]^{+} ;[\alpha]_{\mathrm{D}}^{18}$ : $+24.0^{\circ}(c=0.5, \mathrm{MeOH}) .{ }^{1} \mathrm{H}-\mathrm{NMR}\left(300 \mathrm{MHz}\right.$, pyridine- $\left.d_{5}\right)$ $\delta: 9.84$ (s, H-2), 6.56 (s, H-3), 4.90, 4.77 (each br s, H-30), 3.47 (dt, $J=3.8,11.2 \mathrm{~Hz}, \mathrm{H}-19), 1.77$ (s, H-29). ${ }^{13} \mathrm{C}-\mathrm{NMR}$ (75 MHz, pyridine- $d_{5}$ ) $\delta$ : $191.6(\mathrm{C}-2), 179.3$ (C-28), 164.6 (C-3), 157.7 (C-1), 151.7 (C-20), 110.5 (C-30), 19.9 (C-29).

Determination of Anti-complement Activity through the Classical Pathway Anti-complement activity was determined by modified method of Mayer as described previously. ${ }^{24)}$ For the classical pathway assay, a diluted solution of normal human serum $(80 \mu \mathrm{l})$ collected from a healthy volunteer (Man) was mixed with gelatin veronal buffer $(80 \mu \mathrm{l})$ with or without sample. Each sample was dissolved in DMSO, and used as a negative control. The mixture was preincubated at $37^{\circ} \mathrm{C}$ for $30 \mathrm{~min}$, and sensitized erythrocytes (sheep red blood cells, $40 \mu \mathrm{l}$ ) were added. After incubation under the same conditions, the mixture was centrifuged $\left(4^{\circ} \mathrm{C}, 1500 \mathrm{rpm}\right)$ and the optical density of the supernatant $(100 \mu \mathrm{l})$ measured at $450 \mathrm{~nm}$. Rosmarinic acid and tiliroside were employed as positive controls. The purities of compounds used for the assay were above 95\% checked by HPLC.

\section{RESULTS AND DISCUSSION}

Repeated column chromatography and high performance liquid chromatography (HPLC) of the EtOAc-soluble fraction of $\mathrm{MeOH}$ extract of the fruits of Zizyphus jujuba were performed on a reverse-phase C-18 column, and eleven triterpenoids were isolated (Fig. 1). The structure of the compounds were identified as colubrinic acid (1), ${ }^{10)}$ alphitolic acid (2), 3-O-cis- $p$-coumaroyl alphitolic acid (3), ${ }^{8)}$ 3-Otrans- $p$-coumaroyl alphitolic acid (4), ${ }^{8}$ 3-O-cis- $p$-coumaroyl maslinic acid (5), ${ }^{7)}$ 3-O-trans- $p$-coumaroyl maslinic acid $(6),{ }^{7)}$ betulinic acid (7), oleanolic acid (8), betulonic acid (9), oleanonic acid (10), ${ }^{7)}$ and zizyberenalic acid (11), ${ }^{10)}$ which were elucidated on the basis of their melting points, optical rotations, and spectroscopic data by a comparison with the reported data.

The compounds $(\mathbf{1}-\mathbf{1 1})$ were bioassayed for their classical pathway complement inhibitory activity in vitro following the protocol described earlier (materials and methods section). The result $\left(\mathrm{IC}_{50}\right.$ value) is summarized in Table 1. Of these, compound 5, 6, and $\mathbf{8}$, which are oleanane-type triterpene, had inhibitory effect on the $\mathrm{CP}$ of the complement system with $\mathrm{IC}_{50}$ values of $101.4,143.9$, and $163.4 \mu \mathrm{M}$, respectively. On the other hand, compounds 2, 3, 4, 7, and 9, which are lupan type triterpenes, were inactive. Kapil and Sharma also reported that oleanolic acid inhibited the complement system in vitro and complement-mediated inflammation in vivo. ${ }^{25,26)}$ In view of these results and those previously reported, it appears that oleanolic acid plays a significant role in the anti-complement activity. On the other hand, oleanonic acid (10) was inactive in this assay system when it had a carbonyl group attached to C-3 instead of a hydroxy 


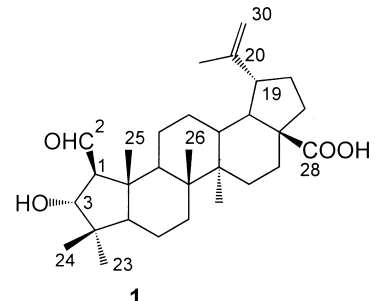

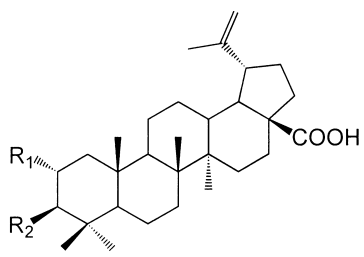

$2 \mathrm{R}_{1}=\mathrm{OH} \quad \mathrm{R}_{2}=\mathrm{OH}$

$3 \quad \mathrm{R}_{1}=\mathrm{OH} \quad \mathrm{R}_{2}=$ cis-p-coumaroyl

$4 \quad \mathrm{R}_{1}=\mathrm{OH} \quad \mathrm{R}_{2}=$ trans- $p$-coumaroyl

$7 \quad \mathrm{R}_{1}=\mathrm{H} \quad \mathrm{R}_{2}=\mathrm{OH}$

$9 \quad R_{1}=H \quad R_{2}==O$

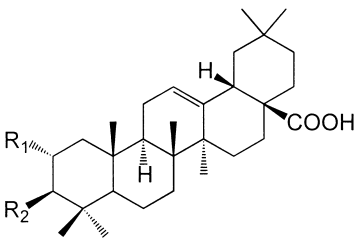

$5 \quad \mathrm{R}_{1}=\mathrm{OH} \quad \mathrm{R}_{2}=$ cis- $p$-coumaroyl

$6 \quad \mathrm{R}_{1}=\mathrm{OH} \quad \mathrm{R}_{2}=$ trans- $p$-coumaroyl

$8 \quad \mathrm{R}_{1}=\mathrm{H} \quad \mathrm{R}_{2}=\mathrm{OH}$

$10 \mathrm{R}_{1}=\mathrm{H} \quad \mathrm{R}_{2}==\mathrm{O}$

p-coumaroyl: $\mathrm{O}-\mathrm{C}-\mathrm{CH}=\mathrm{CH}-\mathrm{OH}$

Fig. 1. Chemical Structures of Compounds from the Fruits of Zizyphus jujuba MiLL

Table 1. Anti-complementary Activity of Triterpenes Isolated from $Z$. jujuba against Complementary System in Vitro

\begin{tabular}{|c|c|}
\hline Compound & $\mathrm{IC}_{50}$ values $(\mu \mathrm{M})^{a)}$ \\
\hline Colubrinic acid (1) & $>200$ \\
\hline Alphitolic acid (2) & $>200$ \\
\hline 3-O-cis-p-Coumaroyl alphitolic acid (3) & $>200$ \\
\hline 3-O-trans-p-Coumaroyl alphitolic acid (4) & $>200$ \\
\hline 3-O-cis-p-Coumaroyl maslinic acid (5) & $101.4 \pm 0.5$ \\
\hline 3-O-trans- $p$-Coumaroyl maslinic acid (6) & $143.9 \pm 0.6$ \\
\hline Betulinic acid (7) & $>200$ \\
\hline Oleanolic acid (8) & $163.4 \pm 0.5$ \\
\hline Betulonic acid (9) & $>200$ \\
\hline Oleanonic acid (10) & $>200$ \\
\hline Zizyberenalic acid (11) & $>200$ \\
\hline Tiliroside $^{b)}$ & 102.2 \\
\hline Romarinic acid $\left.{ }^{b}\right)$ & 180 \\
\hline
\end{tabular}

a) The values represent the mean \pm S.D. of three experiments. b) Used as positive controls. $^{28)}$

group. This suggests that the hydroxy group at C-3 of oleanane-type triterpene may be an important moiety for anti-CP activity, together with the carboxylic acid at C-28, which was known to be an essential feature for this activity. ${ }^{20)}$ However, the carbonyl group at C-3 of the lanostanetype triterpenes, such as ganoderiol F, ganodermanondiol, and ganodermanontriol isolated from Ganoderma lucidum, is an essential structure for the inhibitory activity of the complement system. ${ }^{21)}$

Of the compounds tested, 3-O-cis-p-coumaroyl maslinic acid (5) showed the most potent inhibitory activity against complement-induced hemolysis via the classical pathway. Compared with compound 5 and oleanolic acid (8), compound 5 contained a coumaroyl group at C-3 position on the latter compound. This result suggests that the coumaroyl ana$\log$ at $\mathrm{C}-3$ on the oleanane-type triterpene enhanced the activity compared to that of oleanolic acid. Furthermore, the biacyl sugar analogs at C-3 and 28 of the oleanolic acid (8),

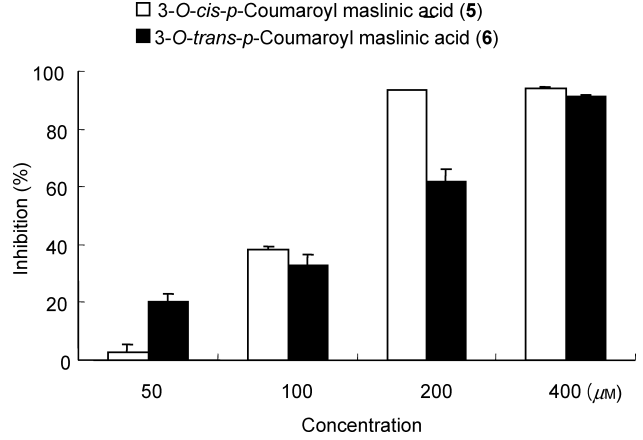

Fig. 2. Inhibitory Effects of 3-O-cis-p-Coumaroyl Maslinic Acid (5), 3-Otrans-p-Coumaroyl Maslinic Acid (6) on Classical Pathway of Complement System (Mean \pm S.D., $n=3$ )

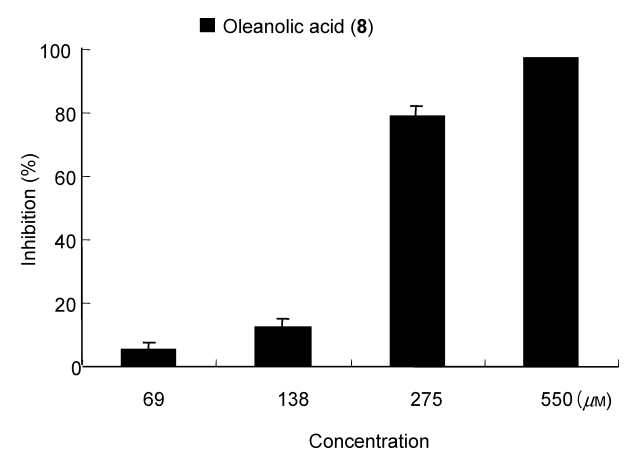

Fig. 3. Inhibitory Effects of Oleanolic Acid (8) on Classical Pathway of Complement System (Mean \pm S.D., $n=3$ )

i.e. ginsenoside Ro, kaikasaponins, soyasaponins, bisdesmosidic oleanolic acids, and HN saponin F, showed complement inhibitory potency superior to oleanolic acid. ${ }^{17-20)}$ In addition, 3-O-trans-p-coumaroyl maslinic acid (6) is a stereoisomer at the coumaroyl group of compoind $\mathbf{5}$ and exhibited lower activity. This indicated that the cis conformation of the coumaroyl group manifested more a potent activity than the trans-form.

This study confirmed that the betulinic acid analogues (compounds 2, 3, 4, 7, and 9) were completely inactive, as was described at previously. ${ }^{27}$ In addition, the ceanothic acid derivatives, colubrinic acid (1) and zizyberenalic acid (11), were also inactive in this assay system, However, 2-hydroxyl3 -coumaroyl-oleanolic acid $(\mathbf{5}, \mathbf{6})$ possessed significant anticomplement activity compared with oleanolic acid (8). Accordingly, oleanolic acid $(\mathbf{8})$ and its coumaroyl analogs $(\mathbf{5}, \mathbf{6})$ might be good candidates as compounds for improving the unwanted and excessive activation of the complement system.

Acknowledgments This research was supported by a grant (PF0300401-00) from the Plant Diversity Research Center of the 21st Century Frontier Research Program funded by the Ministry of Science and Technology of the Korean government and a grant of KRIBB Research Initiative Program (KGS0570412). We are grateful to the Korea Basic Science Institute, Daejeon, Korea, for NMR and mass spectral measurements. 


\section{REFERENCES}

1) Park S. H., Oh S. R., Jung K. Y., Lee I. S., Ahn K. S., Kim J. H., Kim Y. S., Lee J. J., Lee H. K., Chem. Pharm. Bull., 47, 1484-1486 (1999).

2) Lichtman A. K., Pober J. S., "Cellular and Molecular Immunology,” W. B. Sounders Company, Philadelphia, 1997, pp. 315-338.

3) Namba T., "The Encyclopedia of Wakan-Yaku with Color Picture," Vol. I. Hoikusha Co., Osaka, 1993, pp. 142-144.

4) Woo W. S., Kang S. S., Shim S. H., Wagner H., Chari V. M., Seligmann O., Obermeier G., Phytochemistry, 18, 353-355 (1979).

5) Woo W. S., Kang S. S., Wagner H., Seligmann O., Chari V. M., Phytochemistry, 19, 2791-2793 (1980).

6) Naglaa M. N., Food Chemistry, 76, 77-81 (2002).

7) Yagi A., Okamura N., Haraguchi Y., Noda K., Nishioka I., Chem. Pharm. Bull., 26, 3075-3079 (1978).

8) Yagi A., Okamura N., Haraguchi Y., Noda K., Nishioka I., Chem. Pharm. Bull., 26, 1798-1802 (1978).

9) Lee S. S., Lin B. F., Liu K. C., Phytochemistry, 43, 847-851 (1996).

10) Lee S. S., Shy S. N., Liu K. C. S., Phytochemistry, 46, 549-554 (1997).

11) Yoshikawa M., Murakami T., Ikebata A., Wakao S., Murakami N., Matsuda H., Yamahara J., Chem. Pharm. Bull., 45, 1186-1192 (1997).

12) Shou C., Feng Z., Wang J., Zheng X., Planta Med., 68, 799-803 (2002).

13) Su B. N., Cuendet M., Farnsworth N. R., Fong H. H., Pezzuto J. M., Kinghorn A. D., Planta Med., 68, 1125-1128 (2002).

14) Heo H. J., Park Y. J., Suh Y. M., Choi S. J., Kim M. J., Cho H. Y.,
Chang Y. J., Hong B., Kim H. K., Kim E., Kim C. J., Kim B. G., Shin D. H., Biosci. Biotechnol. Biochem., 67, 1284-1291 (2003).

15) Lee S. M., Min B. S., Lee C. G., Kim K. S., Kho Y. H., Planta Med., 69, 1051-1054 (2003).

16) Matsuda H., Murakami T., Ikebata A., Yamahara M., Yoshikawa M., Chem. Pharm. Bull., 47, 1744-1748 (1999).

17) Kim D. S., Oh S. R., Lee I. S., Jung K. Y., Park J. D., Kim S. I., Lee H. K., Phytochemistry, 47, 397-399 (1998).

18) Oh S. R., Kinjo J., Ikeda T., Nohara T., Ahn K. S., Kim J. H., Lee H. K., Planta Med., 66, 506-510 (2000).

19) Park S. H., Oh S. R., Jung K. Y., Lee I. S., Ahn K. S., Kim J. K., Lee J. J., Lee H. K., Arch. Pharm. Res., 22, 428-431 (1999).

20) Oh S. R., Jung K. Y., Son K. H., Park S. H., Lee I. S., Ahn K. S., Lee H. K., Arch. Pharm. Res., 22, 317-319 (1999).

21) Min B. S., Gao J. J., Hattori M., Lee H. K., Kim Y. H., Planta Med., 67, 811-814 (2001).

22) Matsuda H., Tomohiro N., Yoshikawa M., Kubo M., Biol. Pharm. Bull., 21, 1317-1321 (1998).

23) Kundu A. B., Barik B. R., Mondal D. N., Dey A. K., Banerji A., Phytochemistry, 28, 3155-3158 (1989).

24) Min B. S., Lee S. Y., Kim J. H., Lee J. K., Kim T. J., Kim D. H., Kim Y. H., Joung H., Lee H. K., Nakamura N., Miyashiro H., Hattori M., Biol. Pharm. Bull., 26, $1042-1044$ (2003).

25) Kapil A., Sharma S., J. Pharm. Pharmacol., 46, 922 -923 (1994).

26) Kapil A., Sharma S., J. Pharm. Pharmacol., 47, 585-587 (1995).

27) Assefa H., Nimrod A., Walker L., Sindelar R., Bioorg. Med. Chem. Lett., 9, 1889-1894 (1999).

28) Jung K. Y., Oh S. R., Park S. H., Lee I. S., Ahn K. S., Lee J. J., Lee H. K., Biol. Pharm. Bull., 21, 1077-1078 (1998). 